\title{
A deep XMM-Newton serendipitous survey of a middle-latitude area
}

\author{
G. Novara ${ }^{1}$, N. La Palombara ${ }^{1}$, N. Carangelo ${ }^{1,2}$, A. De Luca ${ }^{1}$, \\ P. A. Caraveo ${ }^{1,3}$, R. P. Mignani ${ }^{4}$, and G. F. Bignami ${ }^{3,5,6}$
}

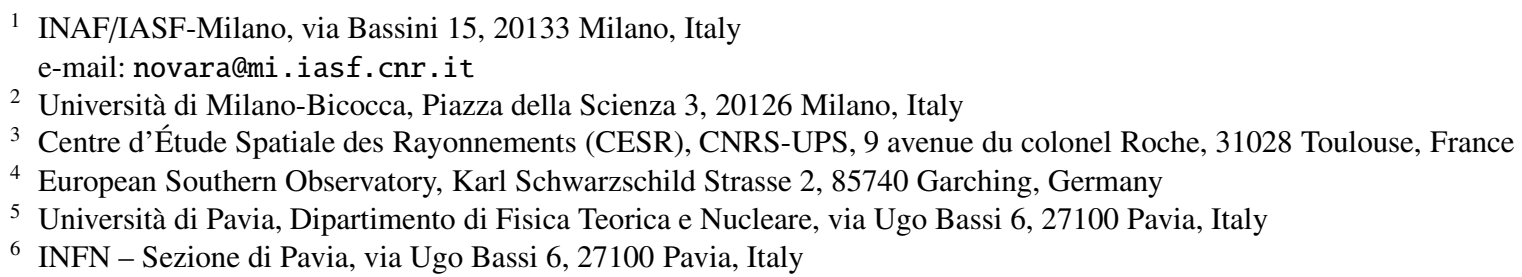

Received 19 May 2005 / Accepted 11 October 2005

\section{ABSTRACT}

The radio-quiet neutron star 1E1207.4-5209 was the target of a $260 \mathrm{ks}$ XMM-Newton observation, that yielded, as a by product, a harvest of about 200 serendipitous X-ray sources above a limiting flux of $2 \times 10^{-15} \mathrm{erg} \mathrm{cm}^{-2} \mathrm{~s}^{-1}$ in the $0.3-8 \mathrm{keV}$ energy range. In view of the intermediate latitude of our field $\left(b \simeq 10^{\circ}\right)$, it comes as no surprise that the $\log N-\log S$ distribution of our serendipitous sources is different from those measured either in the Galactic Plane or at high galactic latitudes. Here we concentrate on analyzing of the brightest sources in our sample, which unveiled a previously unknown Seyfert-2 galaxy.

Key words. galaxies: Seyfert - X-rays: general

\section{Introduction}

The radio-quiet neutron star 1E1207.4-5209 was the target of a 260 ks XMM-Newton observation (De Luca et al. 2004). Such an observation ranges amongst the longest ever performed by $X M M-N e w t o n$, and as of today, it is certainly the longest one at an intermediate galactic latitude (i.e. $|b| \simeq 10^{\circ}$ ).

The deepest X-ray surveys performed, such as the Chandra Deep Field South (Giacconi et al. 2001; Rosati et al. 2002; Giacconi et al. 2002) and North (Brandt et al. 2001), as well as the XMM Lockman Hole survey (Hasinger et al. 2001; Mainieri et al. 2002), encompass only high latitude regions, where serendipitous surveys were also made (Barcons et al. 2002; Della Ceca et al. 2004). On the other hand, X-ray studies of the galactic population have been performed only along the Galactic Plane: shallow, wide-field surveys were obtained by ROSAT (Motch et al. 1998; Morley et al. 2001) and XMM-Newton (Hands et al. 2004), while deep pencil-beam observations of the Galactic Center have been performed by CHANDRA (Muno et al. 2003).

Thus, our long observation at intermediate latitude appears to be well-suited to addressing important issues, such as the ratio between galactic and extragalactic contributors. The combination of the low flux limit, the wide energy band, and the relatively low galactic latitude of this field has the potential for an extremely interesting mix of source types. Owing to the high-energy sensitivity of EPIC, we expect to see through the galactic disk to the distant population of QSOs, AGNs, and normal galaxies. In addition to studying this extragalactic population, our choice of field allows us to sample our Galaxy in great depth. Here again the wide energy range allows both hard and soft sources to be sampled, e.g. a population of X-ray binaries and normal stars.

Characterization of the sources' X-ray spectra, as well as the search for their optical counterparts, are the classical tools to identify our sample of relatively faint sources, either individually or on statistical grounds. Given the range of $f_{\mathrm{X}} / f_{\text {opt }}$ values characteristic of the known classes of X-ray sources (Krautter et al. 1999), we ought to reach $V \simeq 25$ in the optical follow-up to be able to identify the majority of our serendipitous sources. Thus, although useful for a first filtering, Digital Sky Surveys are not deep enough for our purpose and do not provide adequate color coverage.

A proposal for the complete optical coverage of the EPIC field at the $2.2 \mathrm{~m}$ ESO telescope has already been accepted. Waiting for its results, here we outline our detection technique, as well as the global results of such an analysis. Next we focus on analyzing of the brightest sources leading to spectral characterization of a serendipitously discovered Seyfert-2 galaxy. 


\section{X-ray analysis}

\subsection{Observations and data processing}

XMM-Newton observed 1E1207.4-5209 during revolutions 486 and 487 , which resulted in two different pointings separated by $\sim 13 \mathrm{~h}$. All the three EPIC focal plane cameras (Turner et al. 2001; Strüder et al. 2001) were active during both pointings: the two MOS cameras were operated in Full Frame mode, in order to cover the whole field-of-view of 30 arcmin; the $p n$ camera was operated in Small Window mode, where only the on-target CCD is read-out, in order to time tag the photons and provide accurate arrival time information. While the $p n$ data have been used by Bignami et al. (2003) and De Luca et al. (2004) to study the radio-quiet neutron-star 1E1207.4-5209, here we use the MOS data to assess the population of serendipitous sources emerging from this long galactic observation. For both cameras the thin filter was used.

The event files were processed with the version 5.4.1 of the XMM-Newton Science Analysis Software (SAS). After the standard processing pipeline, we looked for periods of high instrument background, due to flares of protons with energies less than a few hundred keV hitting the detector surface. Such soft proton flares enhance the background, so the corresponding time intervals have to be rejected, accordingly reducing the good integration time. In our case, the effective observing time was $\sim 230$ ks over a total observing time of $260 \mathrm{ks}$.

\subsection{Source detection}

In order to maximize the signal-to-noise ratio $(S / N)$ of our serendipitous sources and to reach lower flux limits, we "merged" the data of the two cameras and of the two pointings. We performed the source detection in several energy ranges; first, we considered the two "classical", coarse energy ranges $0.5-2$ and $2-10 \mathrm{keV}$; then, we considered a finer energy division between 0.3 and $8 \mathrm{keV}$, since above $8 \mathrm{keV}$ the instrument effective area decreases rapidly. For each energy band, we generated the field image, the corresponding exposure map (to account for the mirror vignetting), and the relevant background map. The background maps were also corrected pixel by pixel, as described in Baldi et al. (2002), in order to reproduce the local variations.

We also had to take into account that the XMM-Newton image includes a region of diffuse emission characterized by more than 4 events/pixel (Fig. 1), due to the SNR G296.5+10.0. Therefore, we performed the source detection with an "ad hoc" tuning of the parameters inside and outside the SNR area.

The source detection was based on the standard maximum detection likelihood criterium: for each source and each energy range, we calculated a detection likelihood $L=-\ln P$, where $P$ is the probability that the source counts originate from a background fluctuation. We considered a threshold value $L_{\mathrm{th}}=8.5$, corresponding to a probability $P_{\mathrm{th}}=2 \times 10^{-4}$. The actual sky coverage in the various energy ranges was calculated as described in Baldi et al. (2002). In Fig. 2 we show such a coverage for the two coarse energy ranges.
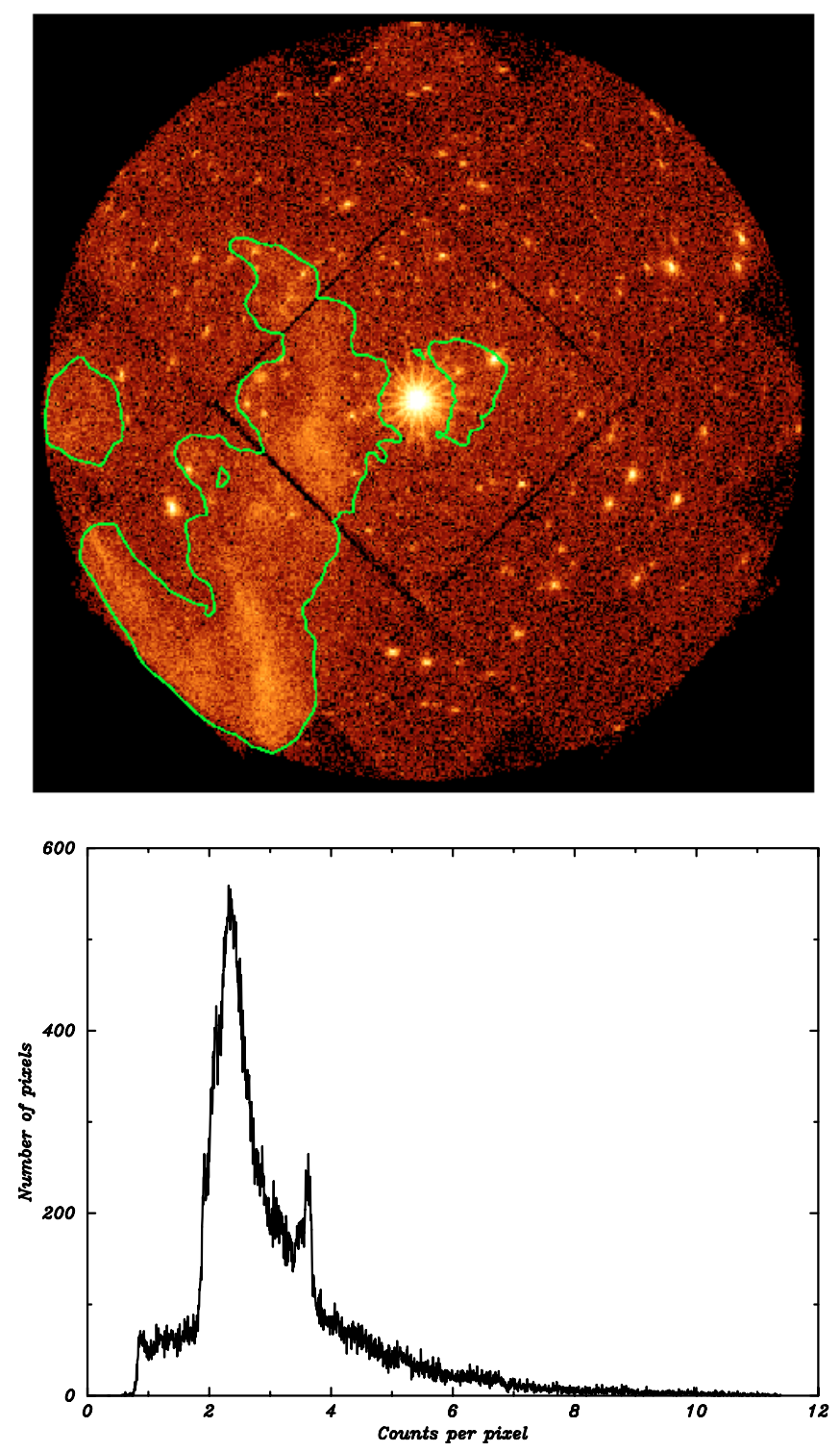

Fig. 1. Top: EPIC MOS image (in the energy range $0.3-8 \mathrm{keV}$ ) of the field of 1E1207.4-5209. Bottom: histogram of the count number per pixel in the background map, in the energy range $0.5-2 \mathrm{keV}$. The sky region corresponding to the tail of the distribution, at values higher than 4 , is enclosed by a green line: it is clearly associated to the area of diffuse emission.

The number of spurious detections in each energy range, obtained by multiplying $P$ times the number of independent (not overlapping) detection cells, is negligible. Indeed, in our detection procedure the area covered by each cell ranges between 0.16 and 0.35 square arcminutes (following the position-dependent Point Spread Function size), so that the $\sim 700$ square arcmin EPIC field-of-view contains at most $5 \times$ $10^{3}$ detection cells. Thus the number of spurious detections is $P_{\text {th }} \times N \leq 1$. Since we performed the source detection in 6 independent energy bands, we expect the total number of spurious detected sources to be at most 6 . When selecting all the sources with $L>8.5$ in at least one of our energy ranges and matching those detected in several energy intervals, we found a total of 196 sources (with a position accuracy of $\sim 5^{\prime \prime}$ ), i.e. 35 inside 

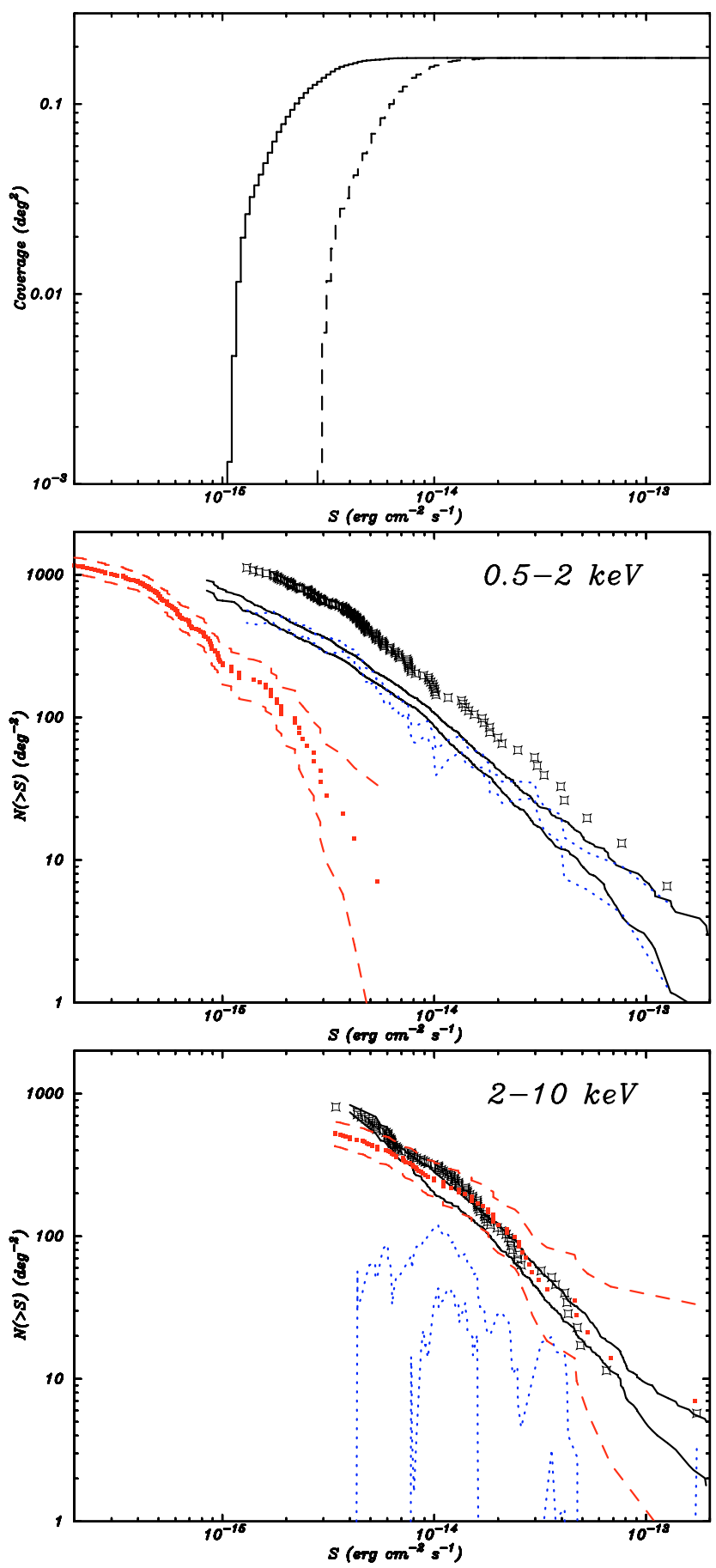

Fig. 2. Sky coverage of the performed observation (top), in the energy ranges $0.5-2 \mathrm{keV}$ (solid line) and 2-10 keV (dashed line), and $\log N-\log S$ distribution of the detected sources (black open squares) in the energy ranges $0.5-2 \mathrm{keV}$ (middle) and $2-10 \mathrm{keV}$ (bottom). The black solid lines trace the upper and lower limits obtained by Baldi et al. (2002) in the same energy ranges but at higher galactic latitudes; the blue dotted lines are the difference between our data and the Baldi et al. ones. The red filled squares and the red dashed lines represent, respectively, the distributions and the limits measured by CHANDRA in the Galactic Plane (Ebisawa et al. 2005).

the area covered by the diffuse emission and 161 outside it. We detected 135 sources between 0.5 and $2 \mathrm{keV}$ and 89 sources between 2 and $10 \mathrm{keV}$, at a flux limit of $1.3 \times 10^{-15}$ and
$3.4 \times 10^{-15} \mathrm{erg} \mathrm{cm}^{-2} \mathrm{~s}^{-1}$, respectively; 68 of them were detected in both energy bands. In order to evaluate the flux of our sources, we assumed a template AGN spectrum, i.e. a powerlaw with photon-index $\Gamma=1.75$ and a hydrogen column density $N_{\mathrm{H}}$ of $1.28 \times 10^{21} \mathrm{~cm}^{-2}$, corresponding to the total galactic column density.

\section{3. $\log N-\log S$ distribution}

In Fig. 2 we show the cumulative $\log N-\log S$ distributions for the sources detected in the two energy ranges. For comparison, we superimposed to our data the lower and upper limits of the $\log N-\log S$ measured by Baldi et al. (2002) for a survey at high galactic latitude $\left(|b|>27^{\circ}\right)$. They obtained the upper limit $\log N-\log S$ by applying the same detection threshold $\left(P_{\text {th }}=2 \times 10^{-4}\right)$ but a larger extraction radius, while the lower $\operatorname{limit} \log N-\log S$ was obtained with the same extraction radius but a more constraining threshold value $\left(P_{\mathrm{th}}=\right.$ $\left.2 \times 10^{-5}\right)$. Moreover, in the same figure we also report the $\log N-\log S$ distributions, as well as the $90 \%$ confidence limits, as measured by CHANDRA in the Galactic Plane (Ebisawa et al. 2005).

In the soft energy band, the $\log N-\log S$ distribution of our sources is well above the high-latitude upper limit, expecially at low X-ray fluxes. Even if the galactic column density represents an overestimate for the stellar population of our sample, we checked that not all of such an excess can be ascribed to overcorrection for the interstellar absorption arising from the use of the total galactic $N_{\mathrm{H}}$ value. We also note that $\sim 60 \%$ of the soft sources were not detected in the hard energy band. In the soft band, the Galactic Plane $\log N-\log S$ distribution (the red points) is much lower than the one at high latitudes, since a significant fraction of extra-galactic sources is not detected. Moreover, the same $\log N-\log S$ is also lower than the difference between our data and the distribution limits at high latitudes (the blue lines). Since Ebisawa et al. (2005) find that most of their soft sources are nearby X-ray active stars, it is possible that our excess over their distribution is due to additional, more distant galactic sources, which are missed looking at $b \sim 0^{\circ}$ but can be detected just outside the Galactic Plane.

In the hard energy band, the distribution of our sources is in good agreement with both the high latitude and the Galactic Plane ones measured by XMM-Newton, CHANDRA, and ASCA (Hands et al. 2004; Ebisawa et al. 2005). At energies $>2 \mathrm{keV}$, we expect the galactic absorption to be negligible so that the extragalactic sources dominate the $\log N-\log S$ distribution at all galactic latitudes, with just a small contribution of the softer galactic sources.

\section{Search for optical counterparts}

In order to identify our serendipitous X-ray sources, we crosscorrelated their positions with two optical catalogues, namely

- version 2.3 of the Guide Star Catalogue (GSC), not yet published, with limiting magnitudes $B_{J} \sim 23$ and $F \sim 22$, photometric accuracy of $\sim 0.25 \mathrm{mag}$ for $B_{J}$ and $\sim 0.2 \mathrm{mag}$ for $F$, and position errors $<0.5^{\prime \prime}$ (Chieregato et al. 2005); 
- the United States Naval Observatory (USNO) catalogue (Monet et al. 2003), with limiting magnitudes $V \sim 21$, $0.2^{\prime \prime}$ astrometric accuracy and $\sim 0.3 \mathrm{mag}$ photometric accuracy.

Six of our X-ray sources have a single bright, almost coincident, optical counterpart. Since the position error is much lower at optical wavelength $\left(\sim 0.5^{\prime \prime}\right)$ than for X-ray $\left(\sim 5^{\prime \prime}\right)$, we used the optical positions to estimate the correction to be applied to the X-ray coordinates. This turns out to be $1.83^{\prime \prime}$ in RA and 1.44" in DEC, for a total of 2.33".

The search for optical counterparts was performed by selecting candidates at $<5^{\prime \prime}$ from the corrected position. In such a way, we found at least one optical candidate counterpart for half of our sources, namely 95 of the 196 sources. Indeed, we found a total of 142 candidate optical counterparts, since for 28 of the $95 \mathrm{X}$-ray sources, we found more than one optical source within the rather conservative $5^{\prime \prime}$ radius error-circle. It is not surprising that half of the detected X-ray sources lack any optical counterpart: in view of the length of our X-ray exposure, the expected limiting magnitude of the possible counterpart is $V \simeq 25$, much lower than the limiting magnitude of the available catalogues. Therefore, the identification of our fainter sources needs ad hoc optical observations that are carried out at ESO.

The above results suggest that we cannot ignore the possible foreground contamination, which could affect our crosscorrelation. The probability of chance coincidence between a X-ray and an optical source is given by $P=1-\mathrm{e}^{-\pi r^{2} \mu}$, where $r$ is the X-ray error-circle radius and $\mu$ the surface density of the optical sources (Severgnini et al. 2005). In our case, within the 15 arcmin radius imaged area, the GSC catalogue provides a total of $\sim 16000$ sources, corresponding to a surface density $\mu \sim 6.4 \times 10^{-3}$ sources $\operatorname{arcsec}^{-2}$. Since the X-ray error-circle is 5 arcsec, we estimated that $P \simeq 0.4$. Therefore up to $40 \%$ of the selected counterparts could be spurious candidates, in rough agreement with the number of X-ray sources with multiple counterparts.

\section{Bright source analysis}

Waiting for the optical data that will allow to characterize our sources on the basis of their $f_{\mathrm{X}} / f_{\text {opt }}$ ratio, we focused on the $\mathrm{X}$-ray analysis of the brightest sources. Since we estimated that at least 500 counts are needed to discriminate thermal spectra from non-thermal ones, we selected sources totalling $>500$ counts. Out of our 196 sources, 24 satisfy this requirement (Fig. 3).

We accumulated the source spectra by selecting only events with PATTERN $=0-12$ and generated ad hoc response matrices and ancillary files using the SAS tasks rmfgen and arfgen. Before spectral fitting, all spectra were binned with a minimum of 30 counts per bin in order to be able to apply the $\chi^{2}$ minimization technique. In this process, the background count rate was rescaled using the ratio of the source and background areas. Then we fitted the source spectra with four spectral models: power-law, bremsstrahlung, black-body,

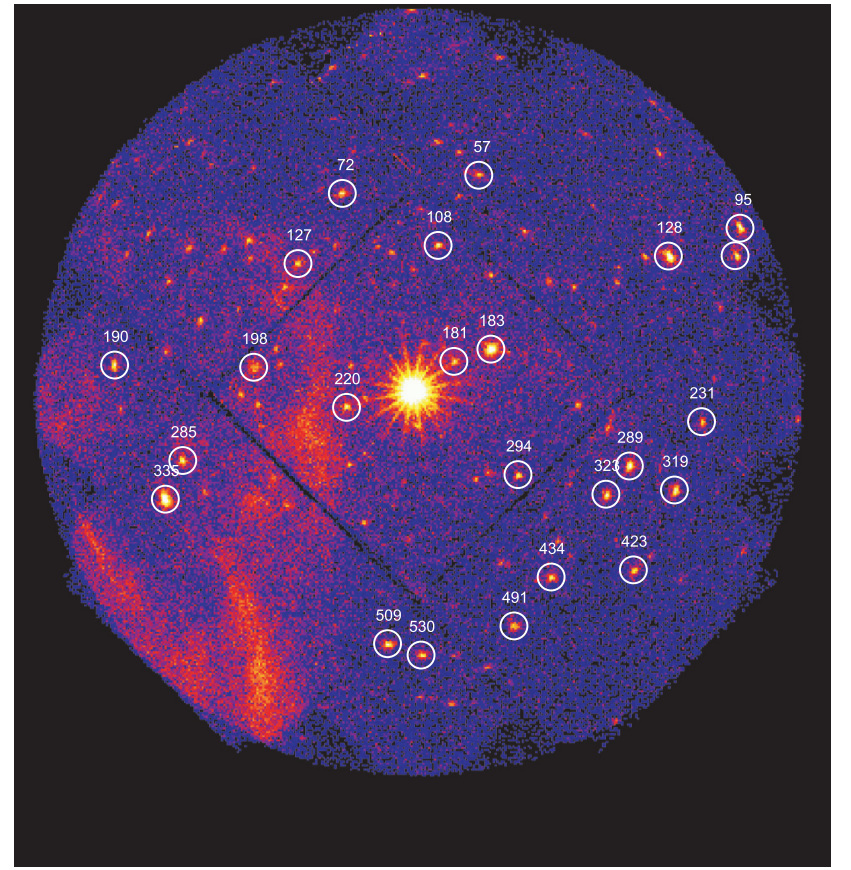

Fig. 3. Image of the sky distribution of the 24 brightest sources, in the energy range $0.3-8 \mathrm{keV}$.

and $m_{e k a l}{ }^{1}$ (i.e. a bremsstrahlung model which includes also the element abundances); in all cases we also included the absorption by the interstellar medium, leaving it as a free parameter. For each emission model, we calculated the $90 \%$ confidence level error on both the hydrogen column density and the temperature/photon-index. In this way we found that 13 sources were best fitted by a power-law model, 2 by a bremsstrahlung model, and 2 by a mekal model (Table 1). For 6 of the 7 remaining sources, at least two different models provided an acceptable fit with a comparable $\chi_{v}^{2}$; finally, for source \#127, all the considered models gave unacceptable results.

The spectral parameters were used to compute the sources' $\mathrm{X}$-ray flux values, to be compared to the optical ones in the framework of the $f_{\mathrm{X}} / f_{\text {opt }}$ identification tool. For the 23 sources with at least one best-fit model, we computed the X-ray flux based on the best-fit values, while for source \#127 we assumed a power-law spectrum with photon-index $\Gamma=1.75$ and a galactic hydrogen column density. On the optical side, we considered all the candidate counterparts found within $5^{\prime \prime}$ radius X-ray error circles. In order to minimize the effect of the interstellar extinction, we used the $F$ magnitude to calculate the source flux, while for the X-ray sources with no counterpart, we used $F=22$ as the optical upper limit.

On the basis of both the spectral fits $\left(N_{\mathrm{H}}\right.$ and best-fit models) and the X-ray-to-optical flux ratios of the possible counterparts, we can propose a firm classification only for 7 sources, i.e. 6 AGNs and 1 star. For 6 additional sources, the suggested classification (i.e. 4 AGNs and 2 stars) is affected by the best-fit value of the interstellar absorption, which is too low

1 Power-low, bremsstrahlung, black-body, and mekal are respectively pow, bremss, bbody, and mekal in XSPEC. 


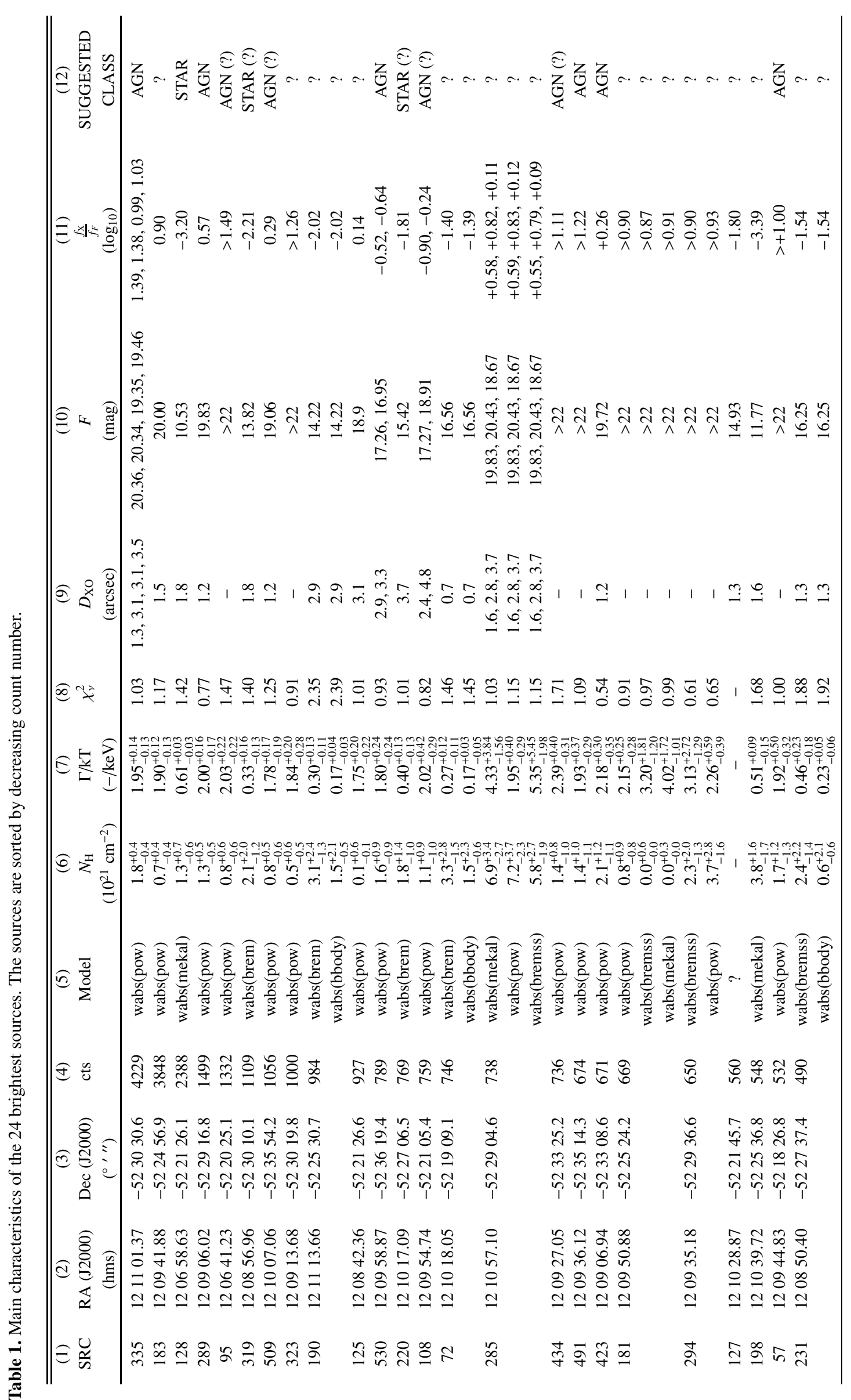

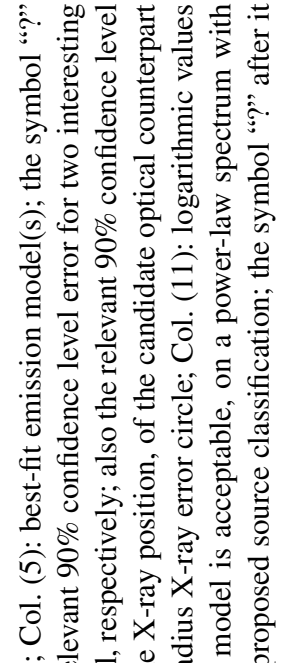

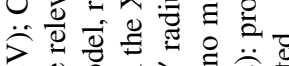

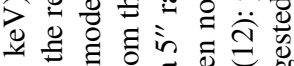

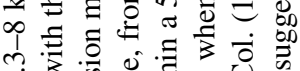

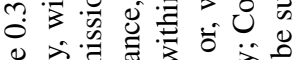

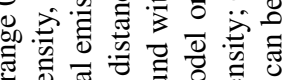
屯ี

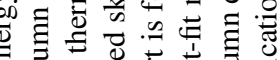
0

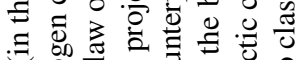

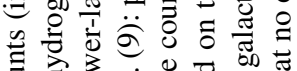

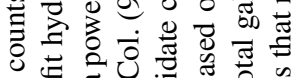

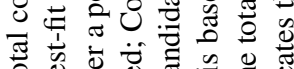

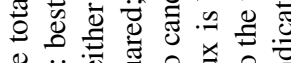

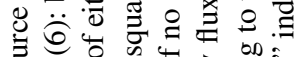
ठํ.

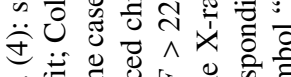

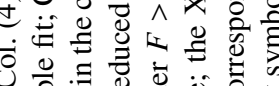

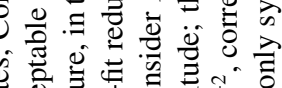

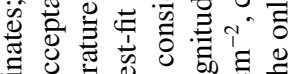

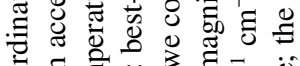

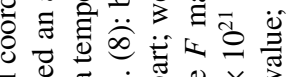

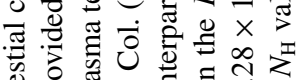

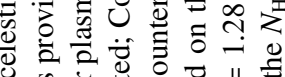
o क

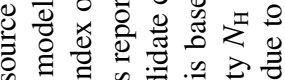

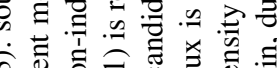

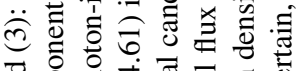

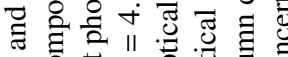

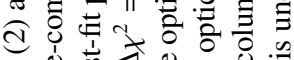

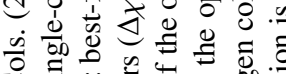
ن. 过

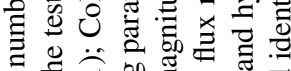

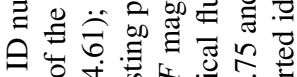

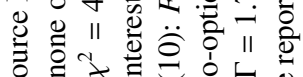

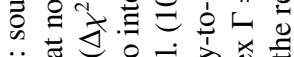

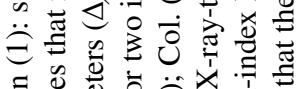

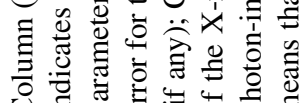




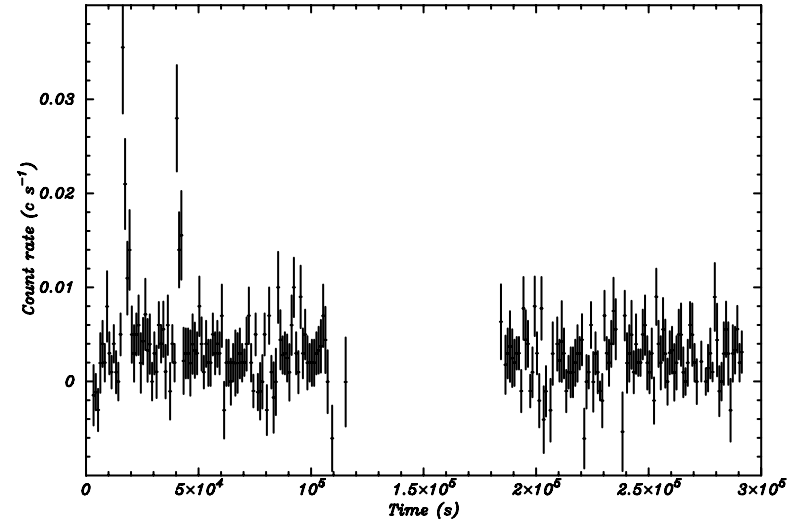

Fig. 4. Light-curve of source \#72, with a 1 ks time binning.

(for AGNs) or too high (for stars) compared to the galactic $N_{\mathrm{H}}$ $\left(1.28 \times 10^{21} \mathrm{~cm}^{-2}\right)$. In view of the large errors on the $N_{\mathrm{H}}$ best-fit values, however, we accept the proposed identification.

Four additional sources (\#190, 72, 198 and 231) are characterized both by a low temperature thermal spectrum and by a low X-ray-to-optical flux ratio, so it is probable that they are stars. Unfortunately they have a high $N_{\mathrm{H}}$ value and, in 3 cases, the emission model is also uncertain; therefore, the star identification cannot be firmly established. For source \#72 this classification would also be supported by the observed light curve (Fig. 4), which shows large but short flares and a flux variability with time-scales of a few hundred seconds.

We note that single component fitting can induce further uncertainty on the $N_{\mathrm{H}}$ estimate. Indeed, stars do show two temperature spectra (actually, coronal loop distributions) which, if fitted with a single temperature, would result in an overestimate of the $N_{\mathrm{H}}$ values. On the other hand, AGNs often have additional soft components that, for a pure power-law fit, would yield $N_{\mathrm{H}}$ values that are too low. In view of the above uncertainties, we underline that the source classification proposed in Table 1 is only tentative.

Only the low $N_{\mathrm{H}}$ value prevents classifying 3 other sources $(\# 183,323$, and 125) as AGNs. They are best fitted by a power-law spectrum with photon index $\simeq 2$ and have a rather high X-ray-to-optical flux ratio. The smooth variability observed for source \#183, with a time-scale of $\sim 10^{4} \mathrm{~s}$ (Fig. 5), would also support an AGN identification ${ }^{2}$.

For 3 sources with hard spectrum (\#285, 181, and 294), it is not possible to distinguish between a power-law and a high temperature thermal emission model. With all models, sources \#285 and 294 show a high $N_{\mathrm{H}}$ value, so they are probably extragalactic objects (either AGNs or clusters of galaxies). On the other hand, in all cases, source \#181 has a very low best-fit value of $N_{\mathrm{H}}$, so it should be a galactic object, even if its nature cannot be established.

Finally, source \#127 has a very unusual spectrum that will be discussed in detail in Sect. 5 .

On the basis of the above results, we conclude that 8 sources out of 23 (i.e. $\sim 35 \%$ ) could belong to the Galaxy.

\footnotetext{
${ }^{2}$ Even if, given the maximum estimated luminosity $\left(L_{\mathrm{X}} \sim\right.$ $\left.10^{32} \mathrm{erg} \mathrm{s}^{-1}\right)$ of a possible galactic counterpart $(6.4 \mathrm{kpc})$, this source could also be a quiescent LMXRB or CV.
}

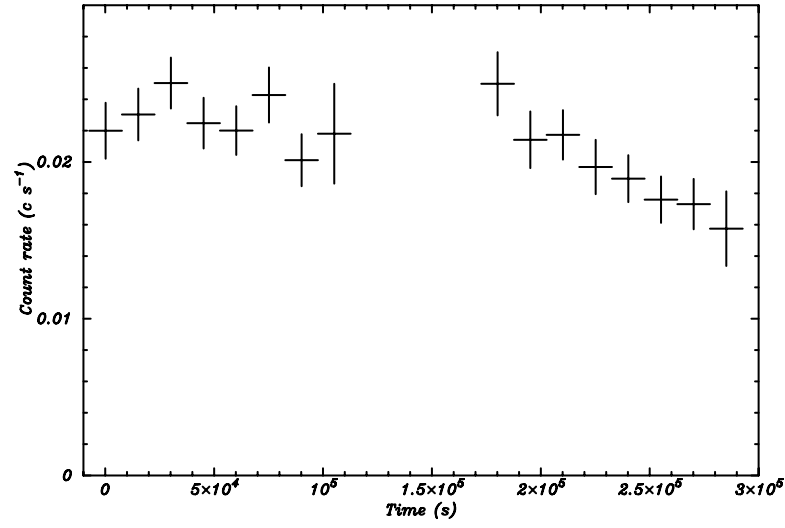

Fig. 5. Light-curve of source \#183, with a $15 \mathrm{ks}$ time binning.

Such a percentage agrees with the results obtained by previous ROSAT surveys that show that the stellar content decreases from $\sim 85 \%$ to $\sim 30 \%$ moving from the Galactic Plane to high galactic latitudes (Motch et al. 1997; Zickgraf et al. 2003).

\section{Source \#127}

The X-ray analysis yields 560 counts in the energy band $0.3-8 \mathrm{keV}$, with a signal-to-noise ratio of 14.64 ; its count rate in the total energy band is $2.03 \times 10^{-3} \mathrm{cts} \mathrm{s}^{-1}$. The source spectrum cannot be described by a standard single-component emission model (Fig. 6), as it is very hard and highly absorbed and is also characterized by a feature at $\sim 6 \mathrm{keV}$, ascribable to an $\mathrm{Fe}$ emission line.

After the astrometric correction, the resulting X-ray position is $\alpha_{\mathrm{J} 2000}=12^{\mathrm{h}} 10^{\mathrm{m}} 28.87^{\mathrm{s}}, \delta_{\mathrm{J} 2000}=-52^{\circ} 21^{\prime} 45.7^{\prime \prime}$. Searching the NED (Nasa/Ipac Extragalactic Database), we found the spiral galaxy ESO 217-G29, located at 1.28" from the X-ray source position. The magnitudes of ESO 217-G29 are $B_{J}=16.74$ and $F=14.93$, and its redshift is $z=$ 0.032 (Visvanathan \& van den Bergh 1992). These parameters, together with the X-ray spectrum and the estimated $\mathrm{X}$-ray-to-optical flux ratio, suggest that source \#127 could be an AGN.

The source is located within the region of diffuse emission (Fig. 1), so its spectrum at low energies $(E<1 \mathrm{keV})$ is polluted by the supernova remnant. Thus we fit the source spectrum only above $1.2 \mathrm{keV}$. According to the AGN unification model (Antonucci 1993; Mushotzky et al. 1993), the source spectrum $S$ has been described by the model

$S=A_{\mathrm{G}}\left[A_{\mathrm{SP}}\left(R_{\mathrm{W}}\right)+A_{\mathrm{T}}\left(P L+R_{\mathrm{C}}+G L\right)\right]^{3}$

where $A_{\mathrm{G}}$ is the galactic absorption $\left(1.28 \times 10^{21} \mathrm{~cm}^{-2}\right), A_{\mathrm{SP}}$ the absorption related to the galaxy hosting the AGN, $R_{\mathrm{W}}$ the warm and optically thin reflection component, $A_{\mathrm{T}}$ the absorption acting on the nuclear emission associated to the torus of dust around the AGN nucleus, $P L$ the primary power-law modeling the nuclear component, $R_{\mathrm{C}}$ the cold and optically thick reflection component, and $G L$ the Gaussian component that

\footnotetext{
3 Wabs*(zwabs*powerlaw + zwabs*(powerlaw + pexrav + zgauss)) in XSPEC.
} 
Table 2. Best-fit parameters for source \#127, for both the optical redshift $z=0.032$ and its best-fit value $z=0.057$.

\begin{tabular}{cccc}
\hline \hline Component & Parameter & $z=0.032$ (fix) & $z=0.057$ \\
\hline$A_{\mathrm{SP}}$ & $N_{\mathrm{H} 1}^{a}$ & $2.26_{-1.10}^{+1.42}$ & $2.39_{-1.14}^{+0.81}$ \\
$R_{\mathrm{W}}$ & $\Gamma$ & 1.9 (fixed) & 1.9 (fixed) \\
& Flux @ $1 \mathrm{keV}^{b}$ & $7.53_{-2.78}^{+3.43}$ & $7.53_{-2.63}^{+3.52}$ \\
$A_{\mathrm{T}}$ & $N_{\mathrm{H} 2}^{a}$ & $75.82_{-19.10}^{+25.02}$ & $82.35_{-24.23}^{+18.69}$ \\
$P L$ & $\Gamma$ & 1.9 (fixed) & 1.9 (fixed) \\
& Flux @ $1 \mathrm{keV}^{c}$ & $1.93_{-0.80}^{+1.37}$ & $1.98_{-0.71}^{+1.51}$ \\
$R_{\mathrm{C}}$ & $\Gamma$ & 1.9 (fixed) & 1.9 (fixed) \\
& Flux @ $1 \mathrm{keV}^{c}$ & $1.93_{-0.80}^{+1.37}$ & $1.98_{-0.71}^{+1.51}$ \\
$G L$ & $E_{\text {line }}(\mathrm{keV})$ & 6.4 (fixed) & 6.4 (fixed) \\
& $I_{\text {line }}^{d}$ & $1.26_{-1.26}^{+1.81}$ & $2.33_{-1.46}^{+2.42}$ \\
& $\mathrm{EQW} \mathrm{(eV)}$ & $185_{-185}^{+265}$ & $311_{-196}^{+322}$ \\
\hline d.o.f. & & 32 & 31 \\
$\chi_{v}^{2}$ & & 1.143 & 1.035 \\
\hline
\end{tabular}

${ }^{a} 10^{22} \mathrm{~cm}^{-2} ;{ }^{b} 10^{-6} \mathrm{ph} \mathrm{cm}^{-2} \mathrm{~s}^{-1} \mathrm{keV}^{-1} ;{ }^{c} 10^{-4} \mathrm{ph} \mathrm{cm}^{-2} \mathrm{~s}^{-1} \mathrm{keV}^{-1}$; ${ }^{d} 10^{-6} \mathrm{ph} \mathrm{cm}^{-2} \mathrm{~s}^{-1}$.

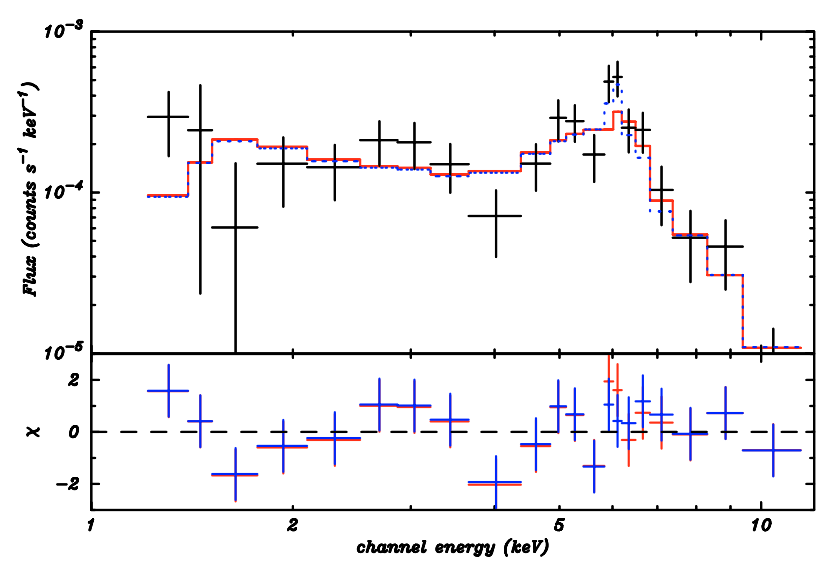

Fig. 6. Top: comparison of the unbinned spectrum of source \#127 with the best-fit model, in the case of both redshift fixed at $z=0.032$ (red solid line) and of best-fit value $z=0.057$ (blue dotted line). Bottom: data-model residuals (in $\sigma$ ) for the two models.

models the Fe line at $6.4 \mathrm{keV}$. For the $A_{\mathrm{SP}}, A_{\mathrm{T}}, R_{\mathrm{C}}$, and $G L$ components, the redshift value is fixed at $z=0.032$ (Visvanathan $\&$ van den Bergh 1992).

The best-fit parameters, listed in Table 2, provide an acceptable fit, yielding $\chi_{v}^{2}=1.143$ with 32 d.o.f.; the value of $N_{\mathrm{H} 2}$ implies that the torus around the AGN is Compton-thin. However, this model does not describe the prominent $\mathrm{Fe}$ line satisfactorily, since it assigns an energy of $6.2 \mathrm{keV}$ to the line centroid (red solid line in Fig. 6), while in the accumulated spectrum the line is centered around $6.0 \mathrm{keV}$; moreover, the line significance is marginal.

Leaving the $z$ value as a free parameter, we obtain a better fit $\left(\chi_{v}^{2}=1.035\right.$ with 31 d.o.f) for $z=0.057_{-0.016}^{+0.009}$, which is quite different to the optical value, although consistent at $2 \sigma$ level; moreover, the line is significant at a $90 \%$ confidence level. Using the F-test, the improvement with respect to the previous fit based on the optical redshift is significant at

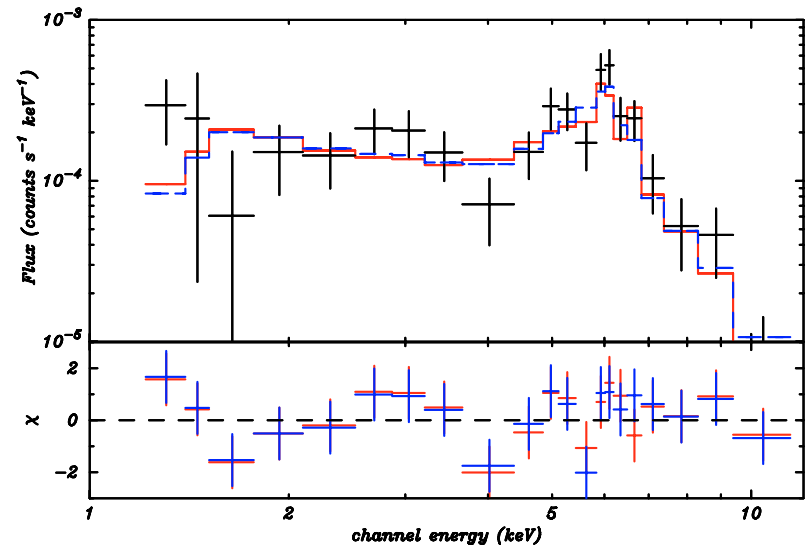

Fig. 7. Top: comparison of the unbinned spectrum of source \#127 with the best-fit model and $z=0.032$, in the case of both a laor (red solid line) and a diskline (blue dotted line) model for the Fe line. Bottom: data-model residuals (in $\sigma$ ) for the two models.

a 95\% confidence level. In Table 2 we report the best-fit parameters of both fits. As a further check, we also applied the Cash statistics to the XSPEC fit and obtained the same results: $z=0.057$ and a normalization of $2.26_{-1.34}^{+2.14}$ for the iron line. If we compare the source spectrum with the best-fit model (blue dotted line in Fig. 6), we note that the Fe line is modelled more accurately and is centered around $6.0 \mathrm{keV}$.

This discrepancy between the X-ray and optical redshift values could be explained by the relativistic broadening of the Fe line. Recently, the rest-frame spectra of several sources detected in the XMM-Newton survey of the Lockman hole showed a relativistically broadened iron line (Streblyanska et al. 2005). Owing to the Compton-thin nature of our source, it is possible that we are observing the same phenomenology. This would explain why the best-fit redshift overcomes the cosmological one. We investigated this possibility by modelling the Fe line with a relativistic line $(R L)$ from an accretion disc. To this aim we replaced the Gaussian component of our model with either a laor (Laor 1991) or a diskline (Fabian et al. 1989) component ${ }^{4}$, leaving $z=0.032$ for the other components. We fixed the emissivity index $\beta$ to 3 and to -2 for the laor and the diskline case, respectively; likewise, in both cases we fixed the line energy to $6.4 \mathrm{keV}$ and the disc inclination angle $i$ to $30^{\circ}$, which is near the best-fit value found by Streblyanska et al. (2005).

In both cases the best-fit model reproduces rather well the Fe line (Fig. 7) and provides an acceptable fit, yielding $\chi_{v}^{2}=$ 1.034 and 1.087 for the laor and the diskline components, respectively. For both models we find that the relativistic component is significant at a $90 \%$ confidence level. However, the disc inner and outer radii values are too small (i.e. a few $R_{\mathrm{g}}$ ) and their difference is not significant. Moreover, only for the laor component is the line EQW comparable to the value of $\sim 0.4 \mathrm{keV}$ found by Streblyanska et al. (2005), while it is significantly larger $(\sim 1 \mathrm{keV})$ for the diskline. Since these parameters are affected by large errors, due to the low count statistics, we conclude that the iron line position can be reconciled with the redshift of the proposed optical counterpart ESO 217-G29.

\footnotetext{
${ }^{4}$ Respectively, laor and diskline in XSPEC.
} 
The 2-10 keV unabsorbed flux of the primary nuclear component is $5.79_{-2.40}^{+4.11} \times 10^{-13} \mathrm{erg} \mathrm{cm}^{-2} \mathrm{~s}^{-1}$ (calculated with $X S P E C)$. Such a flux value, together with the optical magnitude, implies that $f_{\mathrm{X}} / f_{\text {opt }}=0.41$, i.e. well within the AGN range (Krautter et al. 1999). The X-ray luminosity of the source in the $2-10 \mathrm{keV}$ energy band, corrected by the absorption and with the redshift at 0.032 , is $2.59_{-1.07}^{+1.84} \times 10^{42} \mathrm{erg} \mathrm{s}^{-1}$, corresponding to a low luminosity Seyfert galaxy.

Thus, the X-ray spectrum, together with the best-fit value of $N_{\mathrm{H} 2}$ and the nature of the optical candidate counterpart, led us to propose that source \#127 could be a new, low-luminosity Seyfert-2 galaxy discovered serendipitously in our field.

\section{Summary and conclusions}

The longest XMM-Newton observation at low galactic latitude yielded a sample of 135 sources between 0.5 and $2 \mathrm{keV}$ and of 89 sources between 2 and $10 \mathrm{keV}$, with limiting fluxes of $1.3 \times 10^{-15}$ and $3.4 \times 10^{-15} \mathrm{erg} \mathrm{cm}^{-2} \mathrm{~s}^{-1}$, respectively. The $\log N-\log S$ distribution of the hard sources is comparable to the one measured at high galactic latitudes, suggesting that it is dominated by extragalactic sources. On the other hand, the distribution of the soft sources at low fluxes shows an excess above both the Galactic Plane and the high-latitude distributions: we consider this result as a strong indication that we observed a sample of both galactic and extragalactic sources.

We analyzed the 24 brightest sources and proposed an identification for $\sim 80 \%$ of them. Moreover, the detailed spectral investigation of one unidentified source, characterized by a highly absorbed spectrum and an evident $\mathrm{Fe}$ emission line, led us to classify it as a new Seyfert-2 galaxy.

The full X-ray characterization of all the sources, as well as their classification, based on ad hoc optical observations, will be discussed in future papers.

Acknowledgements. We are grateful to K. Ebisawa for providing the $\log N-\log S$ data of the Chandra observation of the Galactic Plane. We wish to thank the referee for useful comments that improved the presentation of our results. We also thank S. Molendi and A. Tiengo for their suggestions and stimulating discussions. This work is based on observations obtained with XMM-Newton, an ESA science mission with instruments and contributions directly funded by ESA Member States and NASA. The XMM-Newton data analysis is supported by the Italian Space Agency (ASI). A.D.L. acknowledges an ASI fellowship. G.N. acknowledges a "G. Petrocchi" fellowship of the Osio Sotto (BG) city council. The Guide Star Catalog used in this work was produced at the Space Telescope Science Institute under a US Government grant. These data are based on photographic data obtained using the Oschin Schmidt Telescope on Palomar Mountain and the UK Schmidt Telescope. This research made use of the USNOFS Image and Catalogue Archive operated by the United States Naval Observatory at the Flagstaff Station (http://www.nofs .navy.mil/data/fchpix/) and of the NASA/IPAC Extragalactic Database (NED), which is operated by the Jet Propulsion Laboratory, California Institute of Technology, under contract with the National Aeronautics and Space Administration.

\section{References}

Antonucci, R. 1993, ARA\&A, 31, 473

Baldi, A., Molendi, S., Comastri, A., et al. 2002, ApJ, 564, 190

Barcons, X., Carrera, F. J., Watson, M. G., et al. 2002, A\&A, 382, 522

Bignami, G. F., Caraveo, P. A., Luca, A. D., \& Mereghetti, S. 2003, Nature, 423, 725

Brandt, W. N., Alexander, D. M., Hornschemeier, A. E., et al. 2001, AJ, 122, 2810

Chieregato, M., Campana, S., Treves, A., et al. 2005, A\&A, 444, 69

De Luca, A., Mereghetti, S., Caraveo, P. A., et al. 2004, A\&A, 418, 625

Della Ceca, R., Maccacaro, T., Caccianiga, A., et al. 2004, A\&A, 428, 383

Ebisawa, K., Tsujimoto, M., Paizis, A., et al. 2005, ApJ, 635, 214

Fabian, A. C., Rees, M. J., Stella, L., \& White, N. E. 1989, MNRAS, 238,729

Giacconi, R., Rosati, P., Tozzi, P., et al. 2001, ApJ, 551, 624

Giacconi, R., Zirm, A., Wang, J., et al. 2002, ApJS, 139, 369

Hands, A. D. P., Warwick, R. S., Watson, M. G., \& Helfand, D. J. 2004, MNRAS, 351, 31

Hasinger, G., Altieri, B., Arnaud, M., et al. 2001, A\&A, 365, L45

Krautter, J., Zickgraf, F.-J., Appenzeller, I., et al. 1999, A\&A, 350, 743

Laor, A. 1991, ApJ, 376, 90

Mainieri, V., Bergeron, J., Hasinger, G., et al. 2002, A\&A, 393, 425

Monet, D. G., Levine, S. E., Canzian, B., et al. 2003, AJ, 125, 984

Morley, J. E., Briggs, K. R., Pye, J. P., et al. 2001, MNRAS, 326, 1161

Motch, C., Guillout, P., Haberl, F., et al. 1997, A\&A, 318, 111

Motch, C., Guillout, P., Haberl, F., et al. 1998, A\&AS, 132, 341

Muno, M. P., Baganoff, F. K., Bautz, M. W., et al. 2003, ApJ, 589, 225

Mushotzky, R. F., Done, C., \& Pounds, K. A. 1993, ARA\&A, 31, 717

Rosati, P., Tozzi, P., Giacconi, R., et al. 2002, ApJ, 566, 667

Severgnini, P., Della Ceca, R., Braito, V., et al. 2005, A\&A, 431, 87

Strüder, L., Briel, U., Dennerl, K., et al. 2001, A\&A, 365, L18

Streblyanska, A., Hasinger, G., Finoguenov, A., et al. 2005, A\&A, 432, 395

Turner, M. J. L., Abbey, A., Arnaud, M., et al. 2001, A\&A, 365, L27

Visvanathan, N., \& van den Bergh, S. 1992, AJ, 103, 1057

Zickgraf, F.-J., Engels, D., Hagen, H.-J., Reimers, D., \& Voges, W. 2003, A\&A, 406, 535 